study of the shunt to assess shunt patency and upper gastrointestinal endoscopy to assess regression of varices.

Results During the study period, 97 patients with $\mathrm{NCPH}$ underwent shunt surgery (proximal splenoadrenal shunt, 8; proximal splenorenal shunt, 74; and interposition mesocaval shunt, 15). Anomalous anatomy of the left renal vein was the main indication (5/8 patients) for a splenoadrenal shunt. Median fall in portal pressure in patients who underwent splenoadrenal shunt was $11.5 \mathrm{mmHg}$ (range, 2-14 mmHg). The median (range) operative time was 4.5 (3-6) hours and median (range) intraoperative blood loss was 160 (100-200) $\mathrm{mL}$. During a median (range) follow-up of 32 (12-48) months, shunt thrombosis developed in one patient. Comparison of intraoperative parameters and postoperative outcomes showed no significant difference in median fall in portal pressure $(p=0.39)$, median operative time $(p=0.51)$, median blood loss $(\mathrm{p}=0.80)$, Grade III/IV postoperative complications $(p=0.56)$, shunt thrombosis $(p=0.93)$, and varices regression rate $(\mathrm{p}=0.72)$ between patients undergoing proximal splenorenal and splenoadrenal shunt.

Conclusions The left adrenal vein is a suitable vascular conduit for porto-systemic shunt surgery. This is especially so if the performance of splenorenal shunt is precluded because of anatomic abnormality of the renal vein.

\section{IDDF2018-ABS-0018 BOERHAAVE'S SYNDROME: A RARE CAUSE OF HYDRO-PNEUMOTHORAX IN NEWBORN}

Deepika Harit*. University College of Medical Sciences and Guru Tegh Bahadur Hospital, Delhi, India

\subsection{6/gutjnl-2018-IDDFabstracts.67}

Background Hydro-pneumothorax following spontaneous esophageal rupture (Boerhaave's Syndrome) is very rare and often fatal. Early diagnosis and treatment of esophageal perforation can be life-saving.

Methods We report a case of a 4 day old term female baby weighing 2800grams. She was admitted with complaints of excessive cry, vomiting, refusal to feed and fast breathing for one day. The baby was exclusively breastfed since birth and was well until one day before admission. On examination, there was severe respiratory distress, cyanosis and pooling of oral secretions. On palpation, crepitus was appreciated on the skin overlying on the right side of the chest wall (suggestive of subcutaneous emphysema). On ausculatation, entire right side of the chest had decreased air entry which raised suspicion of pneumothorax.

Chest X-ray revealed subcutaneous emphysema, right-sided pneumothorax with underlying collapse and orogastric feeding tube was located in the right thoracic cavity (figure 1). Ultrasound chest showed right-sided air with pleural collection with $2 \mathrm{~mm}$ septations. A tube thoracostomy was done on day 2 of admission. Later, the baby was put on a ventilator in view of type I respiratory failure. CECT thorax was done which showed right-sided hydro-pneumothorax with free air pocket in peri-esophageal region in the upper thoracic oesophagus. After giving contrast through ryles tube, free spillage of contrast was seen in right pleural cavity suggestive of rupture of oesophagus.

Results Since the baby was hemodynamically stable, conservative management was done. After 10 days of conservative management, she was referred and transferred to surgical management where the primary repair was done. Follow up after surgery showed clinical improvement.

Conclusions Spontaneous rupture of oesophagus (Boerhaave's Syndrome) in neonates is rare with high mortatlity. Radiological findings include subcutaneous emphysema, hydro pneumothorax, mediastinal/sub diaphragmatic air. The diagnosis is confirmed by the extravasation of contrast material while performing contrast esophagography with or without CT chest.

A conservative non-operative approach is preferred. Surgical closure is by primary repair/resection of the defect, diversion or esophagectomy. This case report aims to create awareness about the importance of early recognition of this life-threatening condition, which if treated timely can be life-saving.

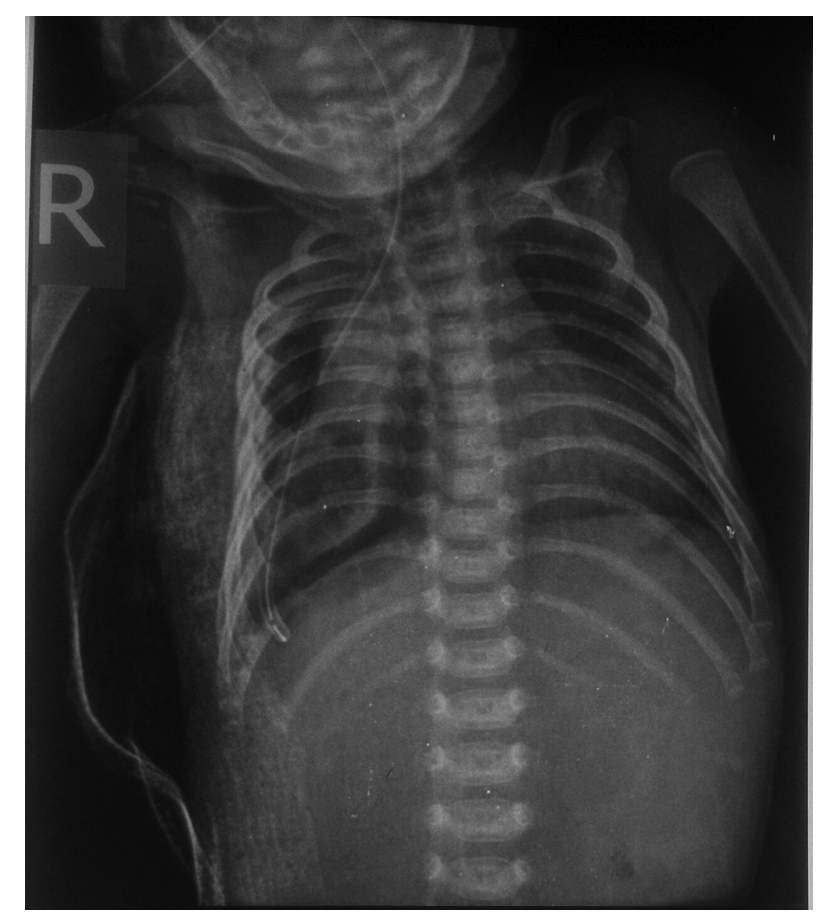

Abstract IDDF2018-ABS-0018 Figure 1

\section{IDDF2018-ABS-0019 ENDOSCOPIC ULTRASOUND-GUIDED FINE- NEEDLE ASPIRATION OF ENLARGED ADRENALS IN PATIENTS WITH PYREXIA OF UNKNOWN ORIGIN: A SINGLE CENTRE EXPERIENCE OF 52 CASES}

Rinkesh Bansal* . Institute of Digestive and Hepatobiliary Sciences, Medanta the Medicity, Gurugram, India

\subsection{6/gutjnl-2018-IDDFabstracts.68}

Background Fine needle aspiration (FNA) of adrenals is needed in patients with pyrexia of unknown origin (PUO) and adrenal enlargement in the absence of other diagnostic clues. Adrenals are easily accessible by endoscopic ultrasound (EUS) due to proximity; however, there is no systemic study available. The aim of this study was to evaluate the diagnostic yield and safety of EUS-FNA of enlarged adrenal in patients with pyrexia of unknown origin (PUO).

Methods Data were analysed from October 2010 to September 2016 at a single tertiary care centre in North India. EUS FNA of enlarged adrenals was done in fifty-two patients for the 
etiological diagnosis of PUO in whom a definitive diagnosis could not be made with other means.

Results The mean age was $48 \pm 14$ years; 36 were males, and 16 were females. EUS-FNA was done from left adrenal in 50 patients and from right adrenal in 2 patients. Technical success was achieved in $100 \%$ cases. The $19 \mathrm{G}$ needle was used in majority $(75 \%)$ due to the presence of necrotic areas in adrenals; median numbers of passes were 2 . The cytopathological diagnoses were tuberculosis $(n=36)$, histoplasmosis $(n=13)$, lymphoma $(n=2)$, and metastasis from undiagnosed neuroendocrine tumour of lung $(n=1)$. Thus a diagnosis could be made in 52/52 (100\%) patients. None of the patients had any procedure-related complications.

Conclusions EUS-FNA is a safe and effective method for evaluating aetiology of PUO in patients with adrenal enlargement.

\section{IDDF2018-ABS-0020 SPECTRUM OF PSEUDOANEURYSMS COMPLICATING ACUTE PANCREATITIS AND ITS RADIOLOGIC MANAGEMENT}

Mukesh Nasa*, Rajesh Puri, Anubhav Khandelwal, Sanjay Saran Baijal. Medanta-The Medicity, India

\subsection{6/gutjnl-2018-IDDFabstracts.69}

Background The aim of the present study was to study the spectrum of pseudoaneurysms secondary to acute pancreatitis, endovascular therapy and outcome of pseudoaneurysms.

Methods Patients with acute pancreatitis with pseudoaneurysm who underwent angiography and coiling of pseudo-aneurysm between Jan 2015 to Jan 2016 were included.

Results Twenty patients of pseudoaneurysms associated with acute pancreatitis were included.

The diagnosis of a pseudoaneurysm was made on computerised tomography (CT) angiography in all the patients.

At angiography, coil embolisation was attempted in 7 patients, glue in 8 patients and coil with glue in 3 patients in the initial sitting. Two patients were taken for percutaneous thrombin occlusion of pseudo-aneurysm, but both had nonobliteration of the pseudo-aneurysm. One underwent coiling, and another had coil along with stent insertion done. There were no episodes of re-bleeding following embolisation. The average size of the pseudo-aneurysm was $8.25 \mathrm{~mm}$. All the patients had single pseudo-aneurysm in the present series.

All patients that were successfully treated demonstrated radiological resolution of their pseudoaneurysms, with a median follow-up of 10 months.

Conclusions Endovascular embolisation is a suitable first-line management strategy for the management of visceral artery pseudoaneurysm complicating acute pancreatitis with low recurrence rates.

\section{IDDF2018-ABS-0021 CAPSULE ENDOSCOPY-INDICATIONS, COMPLETION AND RETENTION RATES- A SINGLE CENTRE EXPERIENCE FROM NORTHERN INDIA}

Mukesh Nasa*, Nisharg Patel, Zubin Sharma, Rajesh Puri. Medanta-The Medicity, India

\subsection{6/gutjnl-2018-IDDFabstracts.70}

Background To study the indications, completion, retention rates and spectrum of lesions on capsule endoscopy
Methods This was a retrospective study of the medical records of patients who underwent capsule endoscopy at our hospital. All patients who underwent capsule endoscopy at our institution for various indications starting from 2012 to 2015 were included in the study.

Results A total of 213 patients underwent Capsule endoscopy for various indications in the study period.

Demographic characteristics: The mean age of the study population was $\underline{59 \text { years, }}$ and male patients were predominant (67\%).

Among the capsule endoscopies done, lesions were identified in $92 \%$ of CE examinations, while normal findings were reported in $8 \%$ of procedures.

The indications for $\mathrm{CE}$ are shown in figure 1.

The common reasons included Obscure GI Bleed (75\%), suspected Crohn's disease (10\%), chronic diarrhoea, abdominal pain, weight loss and Cancer of unknown primary.

\section{Lesions on CE}

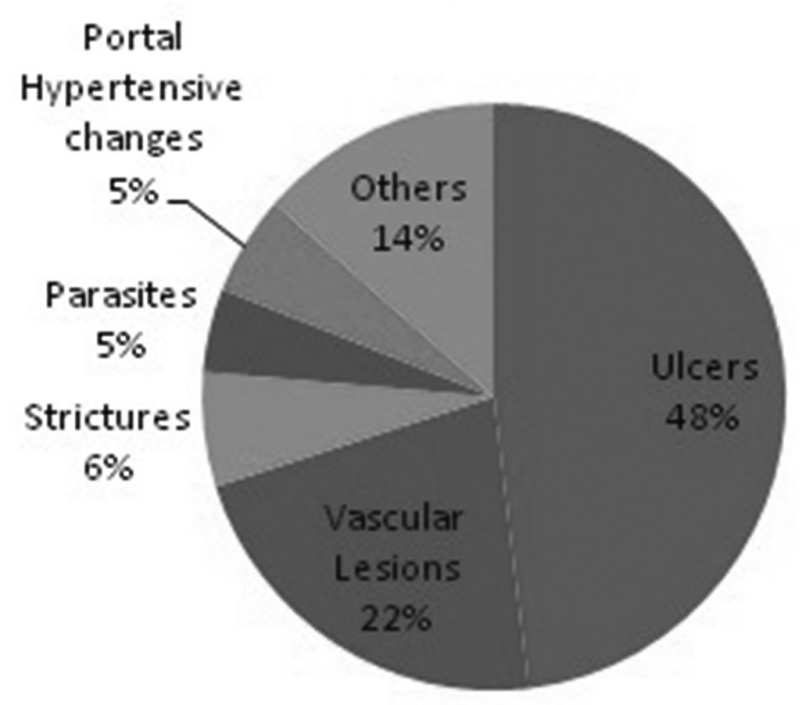

Abstract IDDF2018-ABS-0021 Figure 1 Spectrum of lesions on capsule endoscopy

The most common CE diagnosis was-Small bowel ulcers (48\%),

Vascular Lesions (22\%), and Strictures (6\%) were also common findings.

Conclusions Capsule endoscopy is a safe and excellent modality for evaluation of small bowel.

IDDF2018-ABS-0022 DIAGNOSTIC ADEQUACY AND SAFETY OF ENDOSCOPIC ULTRASOUND GUIDED FINE NEEDLE ASPIRATION IN PATIENTS WITH LYMPHADENOPATHY IN A LARGE COHORTROLE OF ENDOSCOPIC ULTRASOUND (EUS) GUIDED FINE NEEDLE ASPIRATION (FNA) IN PATIENTS WITH LYMPHADENOPATHY IN TERM OF DIA

Sweety Bansal ${ }^{*}$, Rajesh Puri. Institute of Digestive and Hepatobiliary Sciences, Medanta The Medicity, Gurugram, India

10.1136/gutjnl-2018-IDDFabstracts.71 